\title{
Blood Flow during Muscle Contraction and Relaxation in Rhythmic Exercise at Different Intensities
}

\author{
Atsuko KaGAYa and Futoshi OGiTa \\ Research Institute of Physical Fitness, Japan Women's College of Physical Education \\ 8-19-1 Kitakarasuyama, Setagaya-ku, Tokyo 157, Japan
}

\begin{abstract}
Effect of contraction force on blood flow during the contraction and relaxation phase of rhythmic handgrip exercise was studied on 6 healthy women. Velocity of blood flow in the brachial artery and the diameter of the artery were studied by Dopller-ultrasound method. Both the peak and mean velocity of the blood flow were significantly higher during the relaxation period than during contraction, and the velocity during the relaxation period was significantly higher in $30 \% \mathrm{MVC}$ exercise than in $10 \% \mathrm{MVC}$ exercise. However, no significant differences were found in the diameter of the artery between resting and exercise conditions, nor between exercises at different intensities. Thus blood flow during the relaxation phase was significantly increased from $135.7 \pm 18.2 \mathrm{ml} \cdot \mathrm{min}^{-1}(10 \% \mathrm{MVC})$ to $182.5 \pm 19.6 \mathrm{ml} \cdot \mathrm{min}^{-1}(30 \%$ MVC) by an increased contraction force, whereas blood flow during the contraction phase was hardly affected by increased contraction force. (Ann. Physiol. Anthrop. 11(3) : 251-256, 1992)

Key words : Blood velocity, Blood flow, Doppler-ultrasound, Contraction force, Rhythmic handgrip contraction
\end{abstract}

The magnitude of blood flow to working muscle will be a determinant of oxygen uptake in skeletal muscle ( Barclay and Stainsby 1975, Brechue et al 1991, Wagner 1991 ). In rhythmically contracting muscle, blood flow increases during the relaxation period between contractions (Barcroft and Dornhorst 1949, Gault et al 1966, Folkow et al 1970, Wesche 1986, Walle and Wesche 1988 ), whereas, during the contraction period, blood flow will be limited or occluded due to an augmented intramuscular pressure (Sadamoto et al 1983). However, concerning the relationship between contraction intensity and blood flow, conflicting results have been reported. Lind and McNicol (1967) demonstrated that blood flow during contraction increased with contraction force when sustained handgrip exercise was continued below $30 \%$ MVC. On the contrary Byström and Kilbom (1990), and Gaffney et al (1990) showed that the intensity of contraction was not the deciding factor for the resulting contraction blood flow.

The blood flow will change depending on the result of competition among those factors such as intramuscular pressure( Sadamoto et al 1983), muscle sympathetic nervous activity (Saito et al 1986) and metabolic vasodilators (Shepherd 1983). The behavior of these factors during exercise will be different at different work intensities and during different phases of rhythmic contraction. However, due to the technical limitations involved in measuring blood flow non-invasively, very few studies have examined circulation at different phases of rhythmic exercise. Recently a quantitative blood flow measurement in peripheral vessels on a beat by beat basis during rhythmic exercise was made possible using Doller-ultrasound equipment (Wesche 1986, 
Wall $\phi$ e and Wesche 1988, Kiens et al,1989).

The present study was therefore designed to determine the effect of contraction force on blood flow during the contraction and relaxation periods of rhythmic handgrip exercise using Dopplerultrasound equipment for blood flow determination. The effect of contraction force on blood velocity and the diameter of the vessel was also determined.

\section{METHODS}

\section{Subjects}

Six physically active women participated in the study after giving their informed consent. Their age, height and body mass were $21.7 \pm 0.2$ yrs, $157.8 \pm 2.1$ $\mathrm{cm}$ and $53.7 \pm 1.7 \mathrm{~kg}$. All subjects were free of medical problems.

\section{Experimental protocol}

Rhythmic handgrip exercise was performed for 3 minutes in a supine position after a pre-exercise control period. The exercise consisted of $4 \mathrm{~s}$ of contraction followed by $2 \mathrm{~s}$ of relaxation. The loads corresponded to $10 \%$ or $30 \%$ of isometric maximal voluntary contraction (MVC) and they were lifted by $2 \mathrm{~cm}$ when subjects gripped the handle.

\section{Measurement of blood flow}

Brachial artery blood flow was measured at the level of the upper arm using a Doppler-ultrasound apparatus (HP SONOS 1000, Ultrasound imaging system, HP $77030 \mathrm{~A}$ ). A $7.5 \mathrm{MHZ}$ transducer was used for the 2D-mode study and 5.6 $\mathrm{MHZ}$ was used for Doppler-ultrasonic study. The depth of this operating frequency was $4 \mathrm{~cm}$. From the resting period to the first minute into recovery, the ultrasound image, Doppler signals, ECG signals and verbal comments were recorded on a VTR system for later analysis. The ultrasound image was recorded every $30 \mathrm{~s}$ for $5.10 \mathrm{~s}$ by intervening the recording of Doppler signals.

The diameter of the artery was measured on a computer for 5 cardiac cycles every $30 \mathrm{~s}$. The Dop- pler spectrum was studied for 3-5 cardiac cycles during the contraction phase and for 2-3 cardiac cycles during the relaxation phase every $30 \mathrm{~s}$. Peak and mean velocity were measured for each cardiac cycle. Heart rate (HR) was calculated from R-R interval. Blood flow per beat was calculated as $\pi r^{2} V$, where $r$ is radius of the artery and $V$ is integrated velocity of blood flow. Minute volume of blood flow was obtained by multiplying HR and blood flow per beat. Blood flow per beat, and minute volume of flow were calculated for each cardiac cycle, and the values for several cardiac cycles each minute were averaged for each person and each parameter.

The laboratory temperature and relative humidity were kept at $24{ }^{\circ} \mathrm{C}$ and $60 \%$.

\section{Statistical analysis}

Group data are expressed as mean \pm SE. Differences were analyzed by Student's $t$-test for paired samples. A p value less than 0.05 was considered significant.

\section{RESULTS}

Fig 1 shows the diameters of the brachial artery during systolic and diastolic phases. The diameter in systole was a little larger than that in diastole though the differences were not significant. No significant differences were found among diameters of different conditions (rest, contraction and relaxation) and different intensities (10\% MVC vs $30 \%$ MVC).

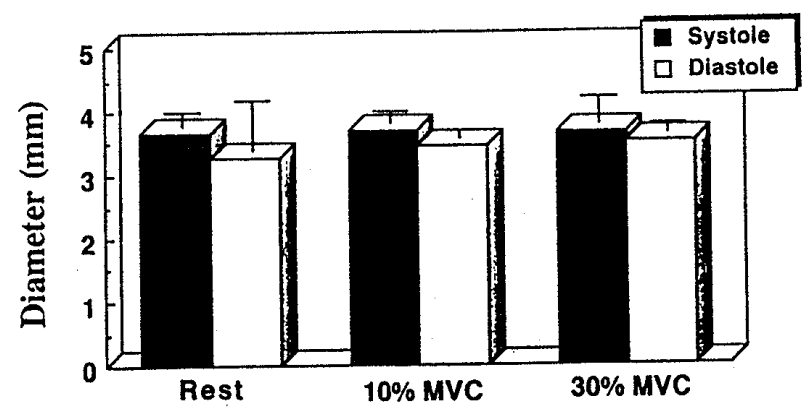

Fig. 1 Diameter of brachial artery at rest and at the end of exercise at $10 \%$ and $30 \%$ MVC (Mean \pm $\mathrm{SE})$. 


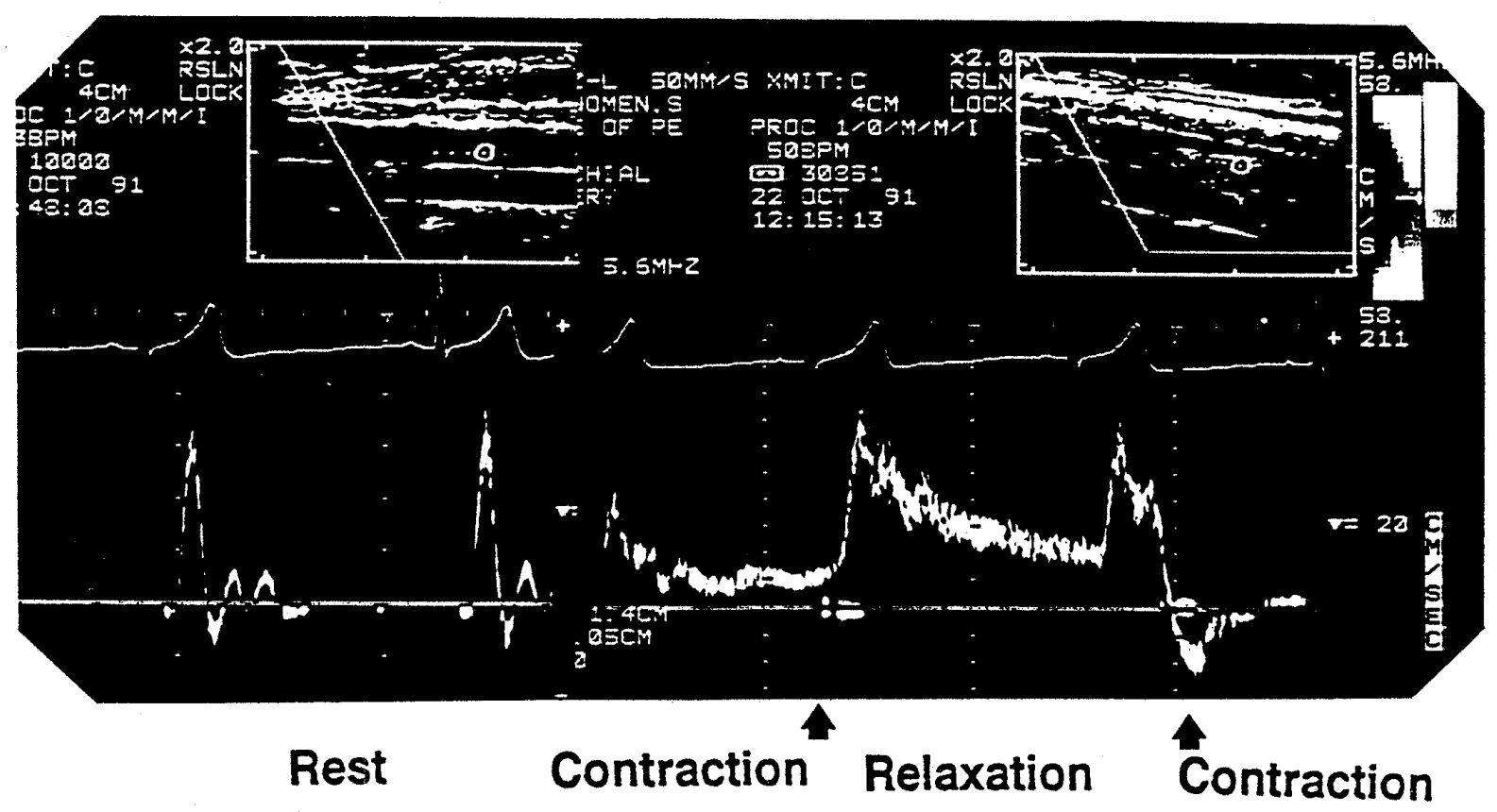

Fig. 2 Doppler spectrum at rest and during exercise at $30 \% \mathrm{MVC}$.

The velocity of blood flow increased during the relaxation phase, and then decreased during the contraction phase (Fig.2). A marked reduction in flow was observed for the first cardiac cycle at the initiation of each muscle contraction followed by recovery to a higher level, which was lower than in the relaxation phase. This first cardiac cycle during the contraction period was excluded from the calculation of velocity and flow.

Peak velocity in each cycle was $79.8 \pm 4.1 \mathrm{~cm} \cdot \mathrm{s}^{-1}$ at rest and increased significantly to $88.9 \pm 4.5 \mathrm{~cm}$. $\mathrm{s}^{-1}(\mathrm{p}<0.01)$ and $90.4 \pm 6.7 \mathrm{~cm} \cdot \mathrm{s}^{-1}(\mathrm{p}<0.01)$ during the contraction phase at the onset of exercise at $10 \% \mathrm{MVC}$ and $30 \% \mathrm{MVC}$ respectively. At the end of $3 \mathrm{~min}$ of exercise, the peak velocity at $10 \% \mathrm{MVC}$ $\left(84.8 \pm 6.6 \mathrm{~cm} \cdot \mathrm{s}^{-1}\right)$ was significantly $(\mathrm{p}<0.01)$ lower than that at $30 \% \mathrm{MVC}\left(103.5 \pm 8.6 \mathrm{~cm} \cdot \mathrm{s}^{-1}\right)$. During the ralaxation period, peak velocity increased to a significantly $(p<0.01)$ higher level than that of the contraction period. There were significant differences between peak velocities during the relaxation period at $10 \% \mathrm{MVC}\left(100.0 \pm 5.64 \mathrm{~cm} \cdot \mathrm{s}^{-1}\right)$ and $30 \%$ MVC $\left(139.7 \pm 7.1 \mathrm{~cm} \cdot \mathrm{s}^{-1}\right)$. Mean velocity for each cardiac cycle is shown in Fig. 3 and was compared between exercises at $10 \% \mathrm{MVC}$ and $30 \% \mathrm{MVC}$.
During the contraction phase, no significant difference was found between intensities except at the end of exercise. During the relaxation phase, however, mean velocity was always significantly higher at $30 \% \mathrm{MVC}$ than $10 \% \mathrm{MVC}$. Mean velocity at the end of exercise at $30 \% \mathrm{MVC}$ reached $76.7 \pm 2.6 \mathrm{~cm}$.
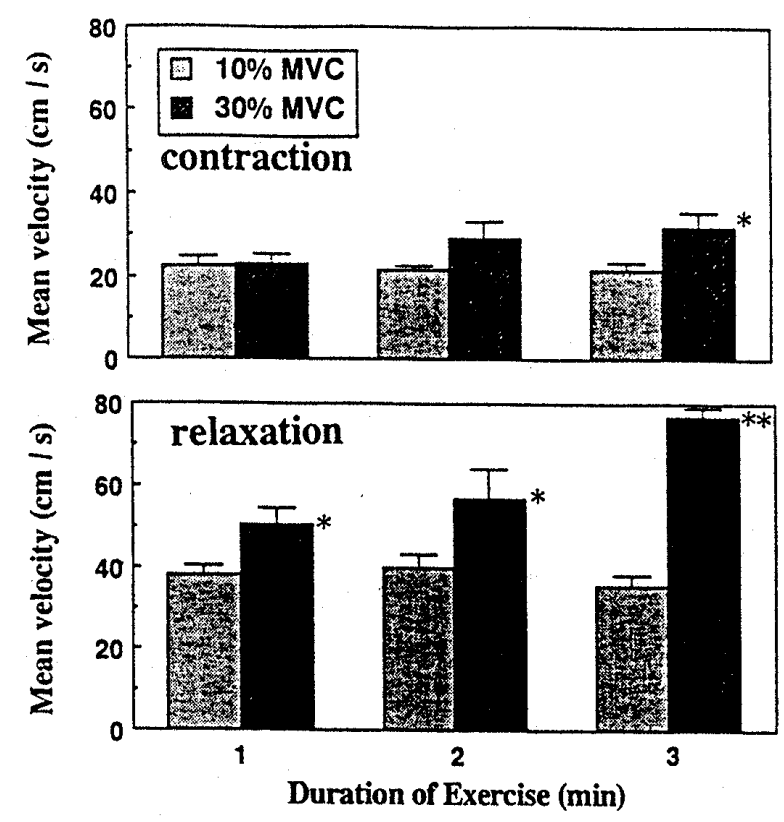

Fig. 3 Mean velocity during the contraction and relaxation period. $(* \mathrm{p}<0.05, * * \mathrm{p}<0.01$; contraction vs relaxation) 


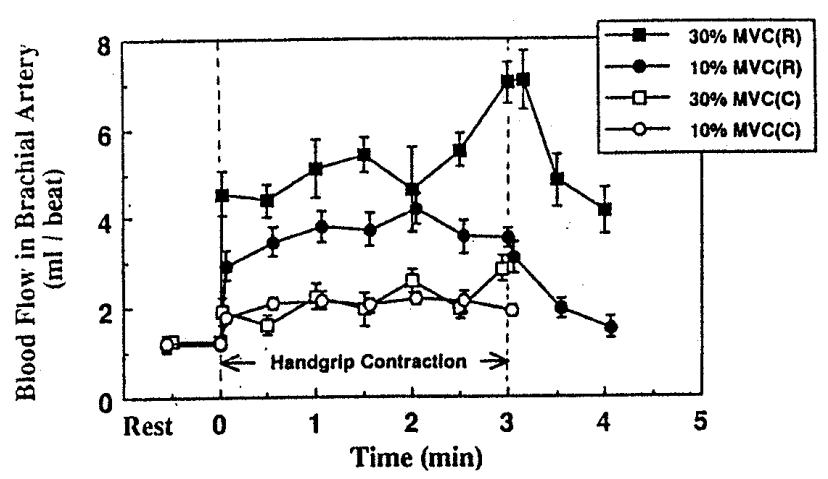

Fig. 4 Blood flow for one cardiac cycle during exercise at different intensities. ( $R$; Relaxation period, $\mathrm{C}$; Contraction period)

$\mathrm{s}^{-1}$ which was significantly higher $(\mathrm{p}<0.01)$ than that $\left(35.3 \pm 3.2 \mathrm{~cm} \cdot \mathrm{s}^{-1}\right)$ at $10 \% \mathrm{MVC}$. The time courses of blood flow changes for one cardiac cycle are illustrated in Fig. 4. At $10 \%$ MVC exercise, blood flow during contraction increased rapidly at the onset of exercise and remained at constant levels for 3 minutes. During the contraction and relaxation period at $30 \% \mathrm{MVC}$ exercise, a marked increase followed a steady flow during first $2 \mathrm{~min}$ utes. Blood flow for one cardiac cycle during the relaxation period was significantly higher than during the contraction at same contraction force. When the magnitude of blood flow was compared between exercises at $10 \% \mathrm{MVC}(3.42 \pm 0.27 \mathrm{ml})$ and $30 \%$ MVC $(7.02 \pm 0.51 \mathrm{ml})$, significant differences were found between blood flows during relaxation phases. During contraction phases, however, no significant difference was observed between blood flows at different intensities except for the mean blood flow upon termination of exercise. Fig. 5 shows that HR was higher during the contraction period and was significantly different between both exercise phases at $30 \%$ MVC. Calculated minute flow at rest was $75.5 \pm 11.0 \mathrm{ml} \cdot \mathrm{min}^{-1}$ before $10 \%$ MVC exercise and $70.7 \pm 7.3 \mathrm{ml} \cdot \mathrm{min}^{-1}$ before $30 \%$ MVC exercise. During exercise, minute flow changed in almost the same manner as did blood flow for one cardiac cycle ( Fig 6) with respect to time course, different phases and different contraction forces. There was no significant difference between

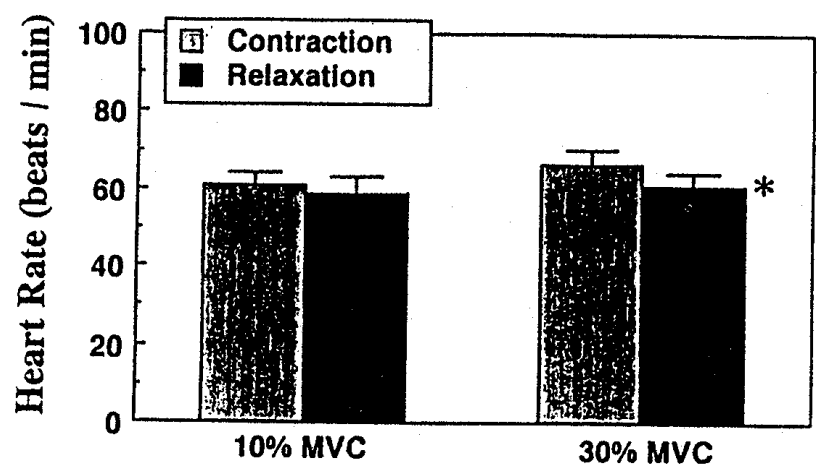

Fig. 5 Heart rate during the contraction and the relax. ation period:

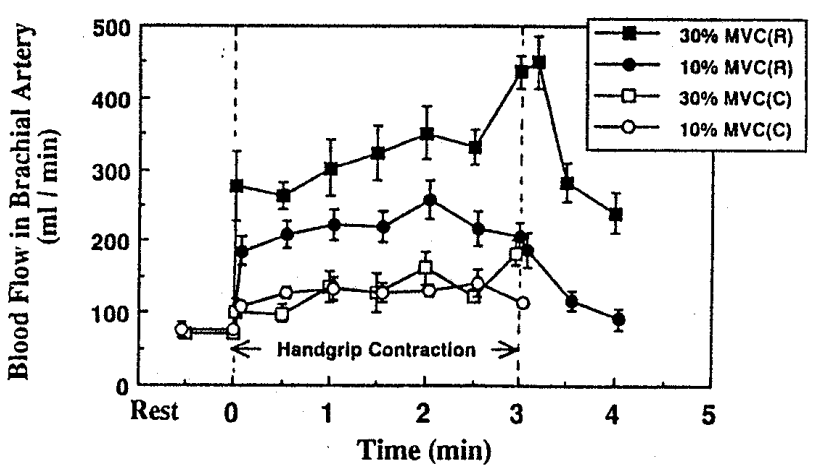

Fig. 6 Minute volume of flow in the brachial artery during the contraction and relaxation period.

blood flow during the contraction period at $10 \%$ MVC $\left(135.7 \pm 18.2 \mathrm{ml} \cdot \mathrm{min}^{-1}\right)$ and $30 \% \mathrm{MVC}(182.5 \pm$ $19.6 \mathrm{ml} \cdot \mathrm{min}^{-1}$ ), while blood flow during the relaxation period was significantly $(p<0.01)$ lower in $10 \%$ MVC exercise $\left(213.0 \pm 16.7 \mathrm{ml} \cdot \mathrm{min}^{-1}\right)$ than that in $30 \% \mathrm{MVC}$ exercise.

\section{DISCUSSION}

The amount of blood flow to active muscle can be increased by accelerating blood velocity and/or by dilating vessel diameter. Ultra-sound apparatus used in the present study enabled us to study these two factors separately. The results of the present study showed that blood velocity increased significantly during graded handgrip exercise, while no significant change was found in the diameter of the brachial artery between rest and during handgrip exercise, nor between exercises at $10 \% \mathrm{MVC}$ and $30 \%$ MVC. Thus, the increased blood flow to the forearm during exercise was explained mainly by 
an acceleration of blood flow.

At the initiation of muscle contraction in the present study, a negative flow was observed for the first cardiac cycle. This negative flow recovered to a level which was a little higher than resting but was lower than during the relaxation phase. Several authors have indicated that the mechanical hindrance of the contraction reduces the blood flow and an increased flow occurs in between contractions ; i.e. relaxation period (Barcroft and Dornhorst 1949, Gault et al. 1966, Folkow et al. 1970, Wesche 1986, Wall $\phi$ e and Wesche 1988). However, the effect of contraction force on the relationship between blood flow during contraction and relaxation has not yet been established. In the present study, the relationship between blood flow during one cardiac cycle and contraction force was investigated. Blood flow during the relaxation period was always higher than during the contraction period. When contraction force increased from $10 \% \mathrm{MVC}$ to $30 \% \mathrm{MVC}$, there was a significant increase in blood flow during the relaxation period, whereas blood flow during the contraction period was not significantly changed except for the the flow upon termination of exercise. This result indicated that the increased rate of oxygen delivery required for contracting muscle at a higher intensity could be met by a corresponding increase in the relaxation flow.

Concerning the effect of contraction force on blood flow during contraction, Lind and McNicol (1967) showed that the forearm blood flow during 3-minute sustained contraction increased with an increase in contraction force within a range of $10-30 \%$ MVC. However, Gaffney et al (1990) indicated that leg blood flow did not increase proportionaly with increasing levels of MVC within 15$50 \%$ MVC. Further, Byström and Kilbom demonstrated from their study on intermittent handgrip exercise that the contraction intensity did not correspond directly to the resulting blood flow. This discrepancy might be explained by the duration of the contraction or the state of fatigue. In the present study, blood flow during contraction at the end of $30 \% \mathrm{MVC}$ was higher than at $10 \% \mathrm{MVC}$, although it did not differ for the first few minutes. Vasodilating metabolites will accumulate if the duration of the contraction is prolonged (Byström and Kilbom 1990).

Blood velocity during the relaxation period increased more at $30 \%$ MVC exercise than during $10 \% \mathrm{MVC}$ exercise, which led to a larger increase in blood flow for one cardiac cycle during $30 \%$ MVC exercise. Peak velocity in the brachial artery increased to $103.5 \mathrm{~cm} \cdot \mathrm{s}^{-1}$ at the end of 3 minutes of exercise at $30 \% \mathrm{MVC}$, which was much lower than the value reported by Wall $\phi$ e and Wesche (1988) for the femoral artery during quadriceps muscle exercise.

A local chemical substance released by contracting muscle (Kiens et al. 1989) and myogenic factors (Wennmalm and Sandgren 1991) associated with the mechanical compression of vessels are considered to be included among possible explanations for a postexercise hyperaemia. In the present study, a forced contraction of $30 \% \mathrm{MVC}$ would cause a mechanical compression of the small veins and elicit a large post-contraction hyperaemia (Wennmalm and Sandgren 1991), which is reflected in the blood flow during relaxation in the present study.

In the present study blood velocity was measured only for several cardiac cycles which started and finished during the contraction phase or relaxation phase. As mentioned-above, backward flow was observed for the first cardiac cycle at the initiation of each contraction, and those cycles were excluded from the calculation. Thus differences in blood flow between contraction and relaxation periods might be underestimated in the present study. Further investigation should be conducted to determine whether blood flow during handgrip contraction at $30 \%$ MVC would reduce arterial inflow below control resting levels as shown in the femoral artery during leg exercise (Wall pe and Wesche 1988).

In summary, blood flow during rhythmic exercise 
was significantly higher during the relaxation period than during the contraction period, even in exercise of lower intensity (10\% MVC). Increased contraction force from $10 \% \mathrm{MVC}$ to $30 \% \mathrm{MVC}$ resulted in an augmented blood flow during the relaxation period, but not during the contraction period of rhythmic exercise of short duration. The increase in blood flow was dependent on the acceleration of blood velocity. The diameter of the artery remained unchanged during exercise at different contraction forces.

\section{Acknowledgement}

The present authors are grateful to Mr. Yukihiro Yamamoto (YHP Co) for his excellent technical assistance and Ms. Donna Gardecki (University of Alberta) for her generosity in improving English.

\section{REFERENCES}

Barclay, J. K. and Stainsby, W. N. 1975 : The role of blood flow in limiting maximal metabolic rate in muscle. Med. Sci. 7 : 116-119.

Barcroft, H. and Dornhorst, A. C. 1949: The blood flow through the human calf during rhythmic exercise. J. Physiol. 109 : 402-411.

Brechue, W. F., Barclay, J. K., O'Dorobinak, D. M., and Stainsby, W. N. 1991 : Differences between $\dot{\mathrm{V}}_{\mathrm{O}_{2}}$ maxima of twitch and tetanic contractions are related to blood flow. J. Appl. Physiol. 71: 131-135.

Byström, S. E. and Kilbom, A. 1990 : Physiological response in the forearm during and after isometric intermittent handgrip. Eur. J. Appl. Physiol. 60: 457-466.

Folkow, B., Gaskell, P. and Waaler, B. A. 1970 : Blood flow through limb muscles during heavy rhythmic exercise. Acta Physiol. Scand. 80: 6172.

Gaffney, F. A., Sjogaard G. and Saltin, B. 1990 : Cardiovascular and metabolic responses to static contraction in man. Acta Physiol. Scand. 138: 249-258.
Gault, J. H., Ross, Jr., J., and Mason, D. T. 1966 : Patterns of brachial artery blood flow in conscious human subjects with and without cardiac dysfunction. Circulation $34: 833-848$.

Kiens, B., Saltin, B., Wall $\phi$ e, L. and Wesche, J. 1989: Temporal relationship between blood flow changes and release of ions and metabolites from muscles upon single weak contractions. Acta Physiol. Scand. 136 : 551-559.

Lind, A. R. and McNicol, G. W. 1967 : Local and central circulatory responses to sustained contractions and the effects of free or restricted arterial inflow on post-exercise hyperaemia. J. Physiol. 192 : 575-593.

Sadamoto, T., Bonde-Petersen, F. and Suzuki, Y. 1983 : Skeletal muscle tension, flow, pressure, and EMG during sustained isometric contractions in humans. Eur. J. Appl. Physiol. 51 : 395-408.

Saito, M., Mano,T., Abe, H. and Iwase, S. 1986 : Responses in muscle sympathetic nerve activity to sustained hand-grip of differennt tension in humans. Eur. J. Appl. Physiol. 55 : 493-498.

Shepherd, J. T. 1983 : Circulation to skeletal muscle. : Handbook of physiology Volume III Section 2. The cardiovascular system (edited by J. T Shepherd and F. M. Abboud) Bethesda, Markland, p319-370.

Wagner, P. D. 1991 : Central and peripheral aspects of oxygen transport and adaptations with exercise. Sports Med. 11 : 133-142.

Wall $\phi$ e, L. and Wesche, J. 1988: Time course and magnitude of blood flow changes in the human quadriceps muscles during and following rhythmic exercise. 405 : 257-273.

Wennmalm, A. and Sandgren, G. 1991: Metabolic and myogenic components of reactive hyperaemia in the human calf. Acta Physiol. Scand. 142: 529-530.

Wesche, J. 1986: The time course and magnitude of blood flow changes in the human quadriceps muscles following isometric contraction. J. Physiol. 377 : 445-462.

(Received March 5, 1992) 